\title{
Análisis estratigráfico de una zona con alto riesgo al deslizamiento por medio de Métodos de Prospección Geofísica
}

\author{
LUIS E. VARGAS \\ Universidad Nacional Autónoma de Honduras, mail: luisfisicaunah@gmail.com
}

Recibido: 17 de Marzo de 2015 / Aceptado: 4 de Mayo de 2015

\begin{abstract}
Resumen
On this work I present results of a research conducted in a zone with high risk to suffer a landslide, located in the neighborhood of Campo Cielo in the town of Comayaguela in Francisco Morazan, Honduras, in February 2013. The research was conducted using the geophysical prospection methods of Seismic Refraction, and Electrical Resistivity. In this study, it was selected as a primary method the Method of Seismic Refraction, since the propopse of the study was to analize the stratigraphic structure of the study area, looking for zones that might be susceptible to have a landslide. The results for this study in comparison with the results obtained with the Electrical Resistivity Method, allow us to identify the zones that are susceptible to have landslides, and at the same time to estimated the thickness of the loss sediment layer, which were estimated at six (6) meters thickness around the crown of the landslide and ten (10) meters in the base of the landslide.
\end{abstract}

Keywords: Stratigraphy, Refraction, Seismic, Kriging, Landslide

En este trabajo se presenta el estudio realizado en una zona con alto riesgo al deslizamiento, ubicada en el Barrio Campo Cielo del municipio de Comayaguela, Francisco Morazán, Honduras; en el período de Febrero de 2013. Dicho estudio se realizó haciendo uso de los Métodos de Prospección Geofísica de Refracción Sísmica, y Resistividad Eléctrica. En este caso en particular, se seleccionó como método primordial el Método de Refracción Sísmica debido a la finalidad del estudio que fue analizar la estructura estratigráfica del lugar, en busqueda de zonas de susceptibilidad a deslizamientos que puedan existir. Con los datos de éste estudio, mediante la comparación de dos métodos aplicados, fue posible determinar zonas de susceptibilidad a deslizamiento que tiene la zona aun, asi como valores estimados del espesor de la capa de sedimento suelto que posee la zona; estimada en seis (6) metros en las cercanias de la corona del deslizamiento y de diez (10) metros en la base del deslizamiento.

Keywords: Estratigrafía, Refracción Sísmica, Kriging, Deslizamiento

\section{INTRODUCCIÓN}

$\mathrm{E}$ L riesgo de una zona a sufrir alguna clase de deslizamiento ha sido de gran importancia para el mundo desde el momento en que como sociedad se empezaron a desarrollar urbanizaciones. Tomando en cuenta siempre el magestuoso poder de la naturaleza en consideracion ante cualquier evento que ésta podría invocar, nos lleva a pensar en formas de prevenir los daños producidos ante dichos eventos. En este caso se consideran los deslizamientos o movimientos de masa de tierra que pueden ser generados por diferentes factores y detonantes naturales. Éste estudio toma como base el análisis de la cantidad de masa que es susceptible a tener un movimiento en un zona de alto riesgo a deslizamientos, en donde previamente han ocurrido estos eventos.

\section{A. Lozalización}

El estudio que se presenta en este artículo fue realizado en el mes de Febrero de 2013, en el barrio Campo Cielo del municipio de Comayaguela, Francisco Morazán, Honduras (ver Figura 1).

\section{B. Impacto del Evento}

El daño sufrido en esta zona se presenta con diferentes caracteristicas como por ejemplo movimientos de masas que desestabilizan el terreno y generan pérdida de soporte en las estructuras, lo que lleva a que estas cedan (ver Figuras 2 y 3), otro daño sufrido se da en la estructura de los servicios de agua potable y de alcantarillado, en donde el movimiento de masas ha llevado a las tuberías a fracturarse, limitando así el servicio que éstas proporcionaban. 


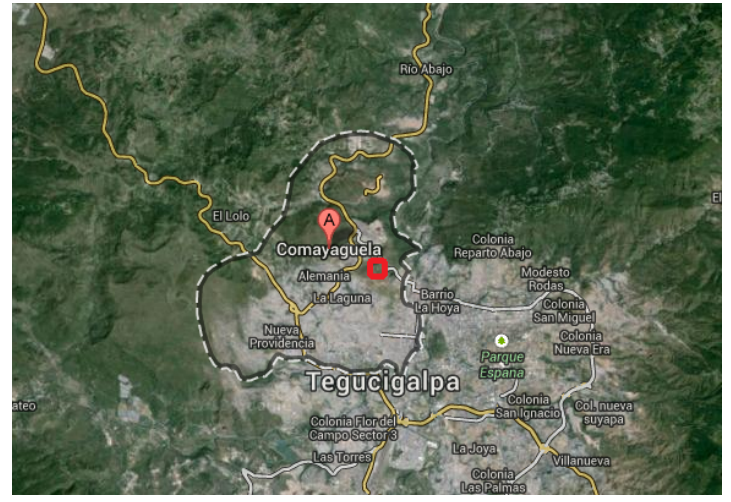

Figura 1: Ubicación de la zona de estudio. El recuadro rojo la ubica dentro del municipio de Comayaguela (Fuente: GoogleMaps).

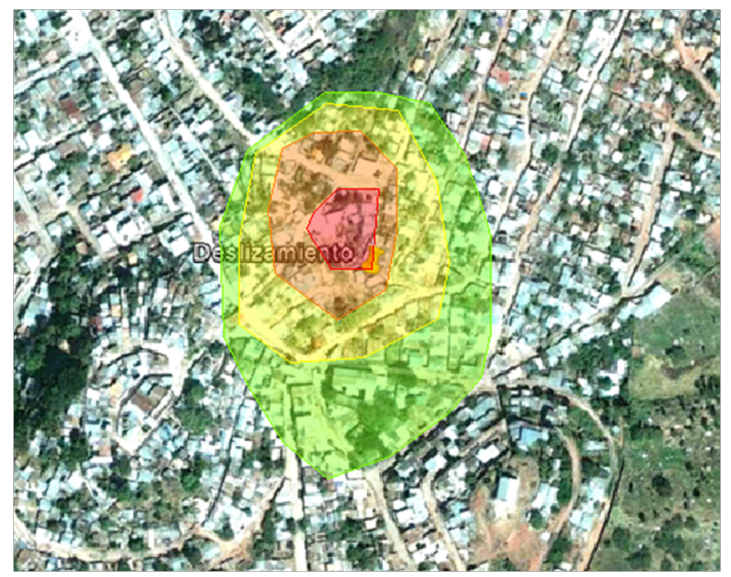

Figura 2: Ilustración de las áreas que sufrieron daño en la zona de estudio, con escala de colores de acuerdo impacto.

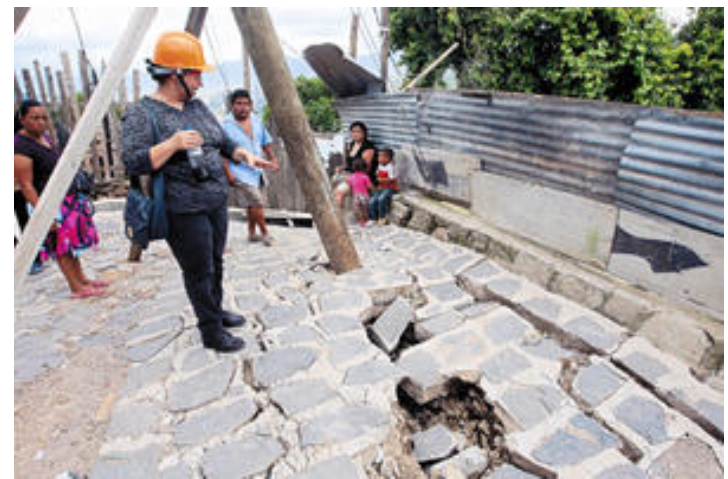

Figura 3: Condiciones de las calles de la zona luego del deslizamiento, presencia de fallas por pérdida de soporte en el suelo.

\section{Finalidad del Estudio}

La finalidad de la investigación fue determinar que áreas dentro de la zona de estudio son susceptibles aun al deslizamiento, considerando la cantidad de sedimentos sueltos que se tienen en toda la zona. El estudio tiene como base el uso de los Métodos de Prospección Geofísica de Refracción Sísmica, que es el método base utilizado para determinar la estratigrafía de la zona y la Tomografía
Eléctrica, que es el método utilizado para corroborar los resultados y validar las hipótesis.

\section{Metodología}

\section{A. Refracción Sísmica}

El Método de Refracción Sísmica es un método de prospección geofísica que consiste en la propagación de una onda artificial u onda de propagación $P$ dentro del medio que se desea estudiar; este como tal, se considera un método de prospección sísmica activa debido a la cantidad de energía necesaria que debe poseer la onda para poderse transmitir por la superficie del suelo y poder recabar toda la información necesaria para el estudio [2.

El método consiste en colocar en línea recta un arreglo de geofonos en la superficie a una separación equidistante entre ellos, estos geofonos son sensores que registran el movimiento de la tierra, que es dado por la onda artificial generada, creando así una señal del tamaño de una pequeña ventana de registro, generalmente de 0.25 segundos. Se registra la señal de cada geofono colocando todos estos juntos para poder observar el fenómeno en cuestion (ver Figura 44.

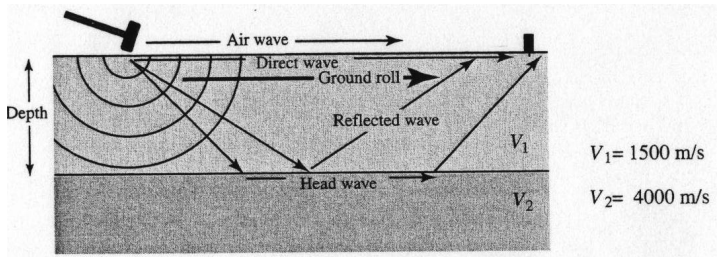

Figura 4: Ilustración del arreglo de los geofonos y como estos reciben la onda artificial de regreso dentro del medio (fuente: [2]).

La onda mecánica que se genera y se transmite a través de la superficie del suelo, una vez que encuentra una superficie de transición entre diferentes tipos de suelos, cambia sus propiedades mecánicas como la velocidad de propagación. Al momento que uno de los rayos del frente de onda entra en contacto con la superficie de transicion, formando el ángulo de incidencia crítico, la onda comienza a actuar con el fenomeno de reflexión interna total, sí la velocidad del segundo medio es mayor a la del primero, entonces la onda se propaga por la superficie de transición, viajando con la velocidad del segundo medio.

En este proceso es cuando se genera la onda frontal que es la que devuelve la onda a la superficie, generando así un cambio en el registro debido al contacto de la onda artificial con la superficie de transición (ver Figura 5 ). Este cambio es el resultado buscado, que nos ayuda a determinar no sólo la velocidad de propagación de la onda mecánica a través de ambos medios sino también nos permite estimar a que profundidad se encuentra esta zona de transición. 


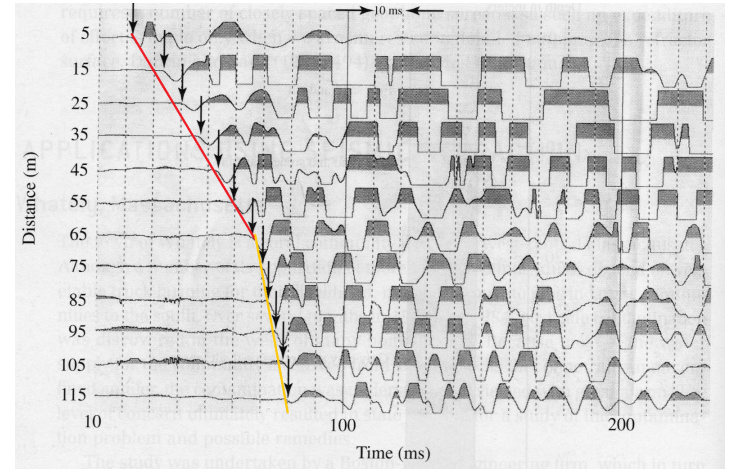

Figura 5: Ilustración del arreglo de registros de cada geofono, en donde se observa el cambio de pendiente debido al arribo de la onda que viajó por la superficie de transición (fuente: [2]).

Las ecuaciones que lideran el análisis de este fenómeno y que proporcionan la base para poder estimar un modelo del medio bajo estudio son las siguientes:

$$
\begin{gathered}
t=\frac{x}{v_{2}}+\frac{2 h_{1} \sqrt{v_{2}^{2}-v_{1}^{2}}}{v_{1} v_{2}} \\
h_{1}=\frac{x_{c o}}{2} \sqrt{\frac{v_{2}-v_{1}}{v_{2}+v_{1}}}
\end{gathered}
$$

En donde $t$ es el tiempo de registro del primer arribo, que es lo que se analiza, basados en el hecho de que éste es el que nos permite observar el cambio de pendiente, que es el dato que se desea obtener. $x$ es la distancia o espaciamiento entre los geofonos, $x_{\text {co }}$ es la distancia de cruce o el punto en donde se observa el cambio de pendiente, $h_{1}$ es el espesor del primer estrato desde la superficie hacia abajo, $v_{1}$ y $v_{2}$ son las velocidades de los estratos, primero y segundo, respectivamente desde la superficie para abajo [2].

Este es el modelo más sencillo para dos estratos, el cual resultó apropiado en ésta investigación debido a que no se buscaba un material en específico, sino observar la diferencia entre material suelto y material más compactado, analizando la relación entre la cantidad de vacíos y la velocidad de propagación de la onda.

\section{B. Modelado: Método de Kriging}

El método de Kriging o proceso de regresión Gaussiana, es un método de interpolación estadístico comúnmente utilizado en geo-estadística, que se basa en una regresión contra los valores observados de $z$ de los alrededores de puntos dados, dándoles peso de acuerdo a valores de covariantes espaciales.

Kriging asigna pesos de acuerdo a la información manejada con una función de pesos, en lugar de una función arbitraria, pero sigue siendo un método de interpolación, por lo que puede mostrar resultados similares a otros métodos. En particular, si la locación de la información es densa y uniformemente distribuida por toda el área de estudio, se obtendrían en este caso buenos resultados con cualquier otro método $[1]$.

Algunas ventajas del método de Kriging son:

- Ayuda a compensar el efecto de la información agrupada, asignándole valores individuales a cada punto con menos peso

- Proporciona un error estimado (varianza de Kriging), junto con los valores estimados de cada variable.

La forma básica del estimador de Kriging es:

$$
Z^{*}(\vec{u})-m(\vec{u})=\sum_{\alpha=1}^{n(\vec{u})} \lambda_{\alpha}\left[Z\left(\vec{u}_{\alpha}\right)-m\left(\vec{u}_{\alpha}\right)\right]
$$

En donde $\vec{u}$ son los vectores de posición, el sub-indice $\alpha$ representa el punto de estimación; $n(\vec{u})$ es el número de puntos a ser determinados; $m(\vec{u})$ son los valores esperados (medias) de las alturas $Z(\vec{u})$ y $Z(\vec{u})_{\alpha}$ y $\lambda_{\alpha}$ son los valores de peso de Kriging que se estiman de forma iterativa al estimar la posición en función de la altura, haciendo uso de un estimador de varianza:

$$
\sigma_{E}^{2}(\vec{u})=\operatorname{Var} Z^{*}(\vec{u})-Z(\vec{u})
$$

\section{Datos o Eventos Seleccionados}

\section{A. Exploración: Selección de Perfiles}

La selección de los perfiles de la zona se basó en las observaciones realizadas a la misma, considerando los daños sufridos debido al movimiento de masa, que debilitó la capacidad de soporte del suelo, lo cual provocó las fallas antes mencionadas (ver Figuras 2 y 3 en la estructura de la urbanizacion.

Los perfiles seleccionados son los mostrados en la figura 6. éstos cubren las zonas de mayor relevacncia para nuestro estudio como: la corona del deslizamiento, el área afectada por la pérdida de soporte y la zona de sedimentanción o base del deslizamiento.

\section{B. Observaciones}

Las observaciones que se tienen de la zona durante el periodo que duraron las mediciones son:

1. Muchas de la calles fallaron, de forma que se logró observar con mayor detalle el suelo de la zona en donde se encontró un alto contenido de material limo arenoso.

2. La mayoría de las áreas se encuentran expuestas a intemperización, lo que debilita el suelo de la zona. 


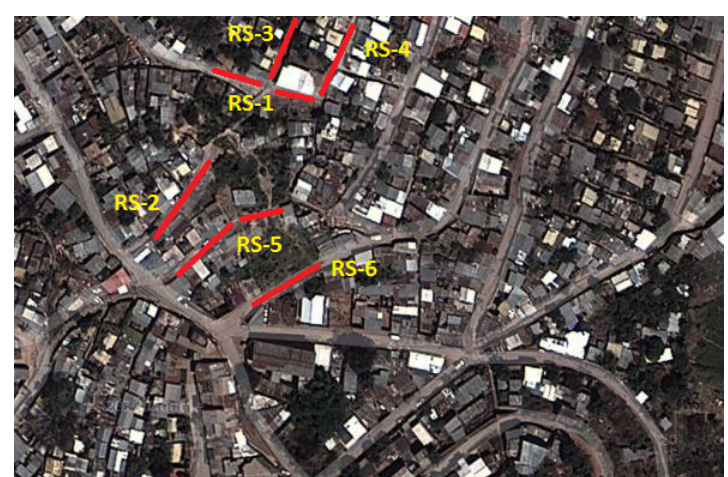

Figura 6: Perfiles seleccionados para el estudio de Refracción Sísmica (fuente: GoogleMpas).

3. En el área donde se produjo el deslizamiento se encontró un alto contenido de vegetación, no correspodiente a la zona para la época en que se realizaron las mediciones, dado que era época de sequía.

Estas, entre otras, observaciones pertinentes de la zona de estudio, nos proveyeron un mejor entendimiento de los modelos desarrollados.

\section{Resultados}

\section{A. Refracción Sísmica}

Todos los resultados obtenidos de los perfiles de Refracción Sísmica son similares al mostrado en la Figura 7. dichos resultados son presentados en el cuadro 1 en donde se puede observar la velocidad de ambos estratos así como el error resultante del modelo propuesto.

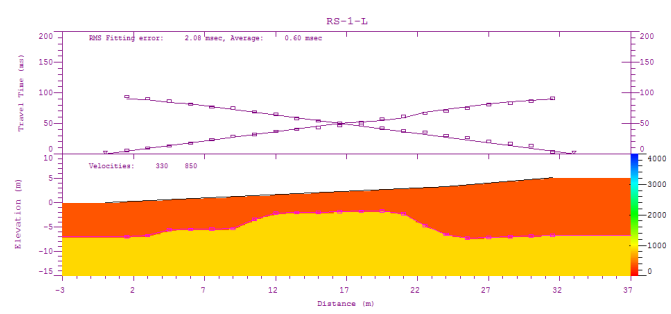

Figura 7: Perfil de Refracción Sísmica de la línea RS-1-L.

\begin{tabular}{|c|c|c|c|}
\hline Perfil & $\begin{array}{c}\text { Velocidad 1 } \\
{[\mathrm{m} / \mathrm{s}]}\end{array}$ & $\begin{array}{c}\text { Velocidad 2 } \\
{[\mathrm{m} / \mathrm{s}]}\end{array}$ & $\begin{array}{c}\text { Error } \\
{[\mathrm{msec}]}\end{array}$ \\
\hline RS-1-L & 330 & 850 & 2.08 \\
\hline RS-1-R & 344 & 979 & 2.97 \\
\hline RS-2-D & 250 & 974 & 1.60 \\
\hline RS-3-U & 317 & 682 & 8.26 \\
\hline RS-4-U & 323 & 659 & 7.64 \\
\hline RS-5-L & 344 & 786 & 1.65 \\
\hline RS-5-D & 309 & 619 & 4.26 \\
\hline RS-6-L & 360 & 924 & 3.79 \\
\hline
\end{tabular}

Cuadro 1: Resultados obtenidos de los perfiles realizados por Refracción Sísmica.
Estos resultados muestran un bajo nivel de error, por lo que se considera que los modelos utilizados fueron adecuados.

\section{B. Modelo 3D Capa Inferior de Sedimento}

Los resultados obtenidos de los perfiles de refracción sísmica, proveen una idea de como es la estratigrafía de la zona en un punto en específico, considerando la cobertura manejada por la cantidad de perfiles realizados, así como la suposición del principio de horizontalidad de los estratos, podemos inferior la realización de un modelo aproximado en 3D de como sería la superficie de la capa inferior de sedimento (ver Figura 8). Dicho modelo se realizó haciendo uso del método de Kriging [1].

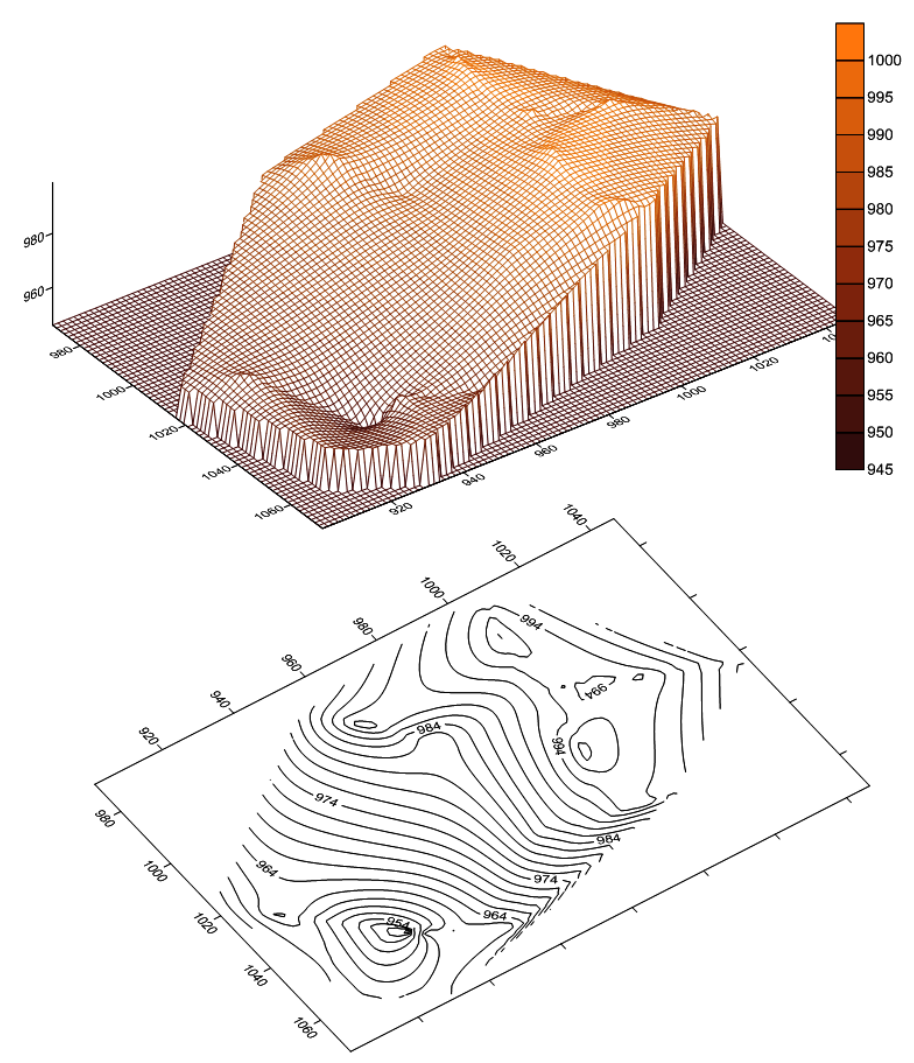

Figura 8: Perfiles seleccionados para el estudio de Refracción Sísmica (fuente: GoogleMpas).

\section{Resistividad Eléctrica}

Al ser un estudio de prospección geofísica, que es un método de mediciones indirectas, siempre debe existir una forma de validarlo, en este caso se escogió realizar como método de validación el método de tomografía eléctrica que mide un perfil de resistividad electrica del suelo y permite determinar diferentes caracteristicas del mismo, considerando los valores de resistividad medidos [3].

Los perfiles de resistividad eléctrica presentan resultados en cuanto a estratigrafía que son similares en cuanto a profundidad a los de refracción sísmica. 


\section{Discusión}

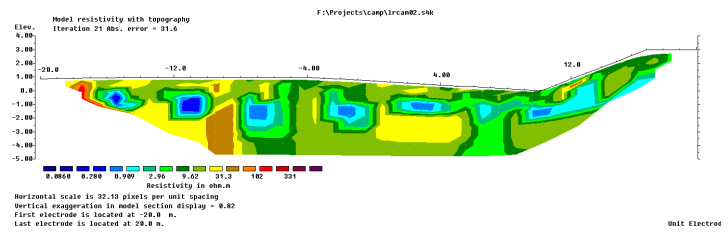

Figura 9: Perfil de resitividad eléctrica paralelo al perfil de Refracción Sísmica $R S-2-D$

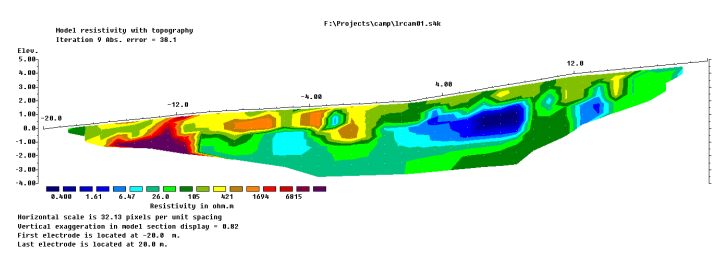

Figura 10: Perfil de resitividad eléctrica paralelo al perfil de Refracción Sismica $R S$-3-U

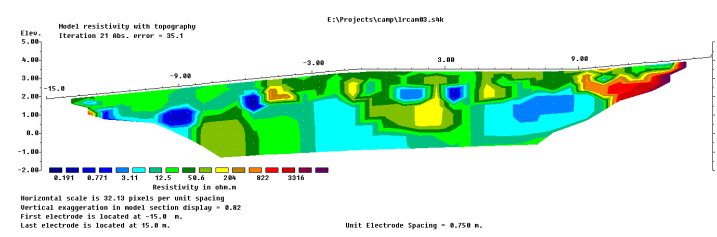

Figura 11: Perfil de resitividad eléctrica paralelo al perfil de Refracción Sísmica RS-5-L

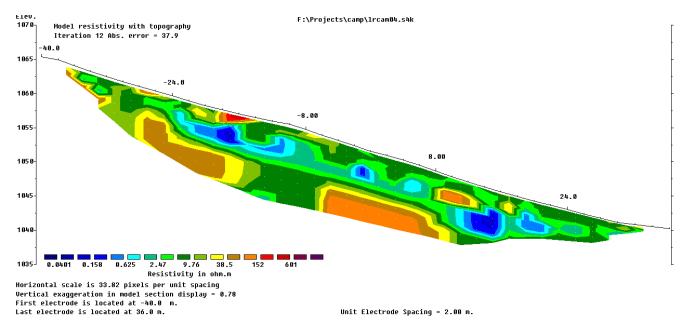

Figura 12: Perfil de resitividad electrica paralelo a la línea del deslizamiento
Al comparar ambos resultados se ha logrado encontrar las concordancias entre los modelos propuestos por ambos métodos, como por ejemplo se puede mencionar que la suposición de manejar sólo dos tipos de estratos no estaba lejos de la verdad, tomando en cuenta que el sedimento suelto tendría un valor más alto de resistividad eléctrica al considerar el modelo trifasico del suelo, en donde mayor cantidad de vacios en el medio proporciona un valor alto de aire en el modelo, el cual posee mayor resistividad eléctrica.

Se debe mencionar, como se puede notar en los resultados de resistividad eléctrica, que no solo se encontró diferencia de material, así mismo aparecen dentro de la tomografía puntos de afluentes de agua o puntos de baja resistividad eléctrica, los cuales concuerdan con las observaciones realizadas en la zona ("Alto contenido de vegetacion en las cercanías de la zona de deslizamiento, algo no concordante con la epoca de sequía") resultados que son concluyentes sobre la presencia de puntos de afluentes de agua en la zona.

Estos puntos de afluentes de agua pueden ser considerados como puntos donde existen incrementos de subpresión debido a la cantidad de agua que circula por los vacíos del sedimento suelto.

Al tomar en cuenta ambos detalles se puede inferir que estos puntos de afluentes de agua pueden considerarse como futuros detonantes de otro deslizamiento al acercarse la época lluviosa en la región, en donde una vez recargado el acuífero del área, aumentarían los esfuerzos de subpresión, disminuyendo así la capacidad de soporte del suelo debida a la fricción interna del material, lo que conllevaría a activar nuevamente el deslizamiento de la zona.

En especial se deben considerar los esfuerzos que se encuentran en la dirección del deslizamiento (ver Figura 12, en donde estos esfuerzos de subpresión, al estar localizados en la zona del deslizamiento podrian causar con menor recarga una nueva detonación del deslizamiento.

Tomando en cuenta el modelo obtenido por el método de Kriging, haciendo uso de los resultados del estudio de refracción sísmica de la zona, se determinó una cota del estrato de sedimento suelto de alrededor de seis (6) metros para la zona cercana a la corona del deslizamiento y de diez (10) metros en la zona baja del deslizamiento. Estos resultados se validan al compararlos con los resultados obtenidos del estudio de resistividad eléctrica, el cual proporcionó patrones similares de tipos de suelo, en función de la resistividad eléctrica del suelo.

\section{Conclusiones}

Se logró identificar la zona que es más susceptible a un nuevo deslizamiento de masas de suelo basados en la cantidad la proyección de las curvas de nivel observas del modelo de Kriging, generado con los resultados de refracción sísmica, así como el análisis de los resultados de 
resistividad eléctrica; los cuales proporcionaron la ubicación de los puntos más susceptibles debido a los esfuerzos de subpresión.

Así mismo se logró determinar un espesor promedio para el sedimento suelto de alrededor de seis (6) metros en la zona cercana a la corona del deslizamiento y de diez (10) metros en la base de la zona del deslizamiento, lo que nos lleva a dar una idea de la estrutura estratigráfica del subsuelo en la zona con alto riesgo al deslizamiento.

\section{Agradecimientos}

Un agradecimiento al Instituto Hondureño de Ciencias de la Tierra, que sin su ayuda y apoyo con el equipo y materiales para realizar las mediciones, este trabajo no se habría realizado. Así mismo un agradecimiento al M.Sc. Manuel Rodriguez, que sus consejos y guía siempre le será agradecida en la formación académica de su servidor.

\section{REFERENCIAS}

[1] G. Bohling. Kriging, 2005.

[2] Sheehan A. F. y Jones C. H. Burger, H. R. Introduction to applied geophysics. 2006.

[3] Sheehan A. F. y Jones C. H. Burger, H. R. Introduction to applied geophysics. 2006. 